\begin{tabular}{|l|r|r|r|r|}
\hline Cuadernos I. Geográfica & $18-19$ & pp. 95-110 & Logroño & 1992-93 \\
\hline
\end{tabular}

\title{
CAPITAL MULTINACIONAL Y CRISIS TEXTIL EN LA RIOJA
}

E. A. CLIMENT LOPEZ*

\begin{abstract}
RESUMEN. Desde los años finales de la década de los ochenta se está produciendo en toda España una aguda crisis de la industria textil, a la que no escapan las empresas instaladas en La Rioja. El acontecimiento más destacado de la misma en la región ha sido el cierre de dos empresas de un grupo multinacional. El artículo describe la trayectoria de dichas empresas, relacionándola con la evolución general de la industria textil riojana e insistiendo fundamentalmente en la situación reciente, y se analiza la estrategia de respuesta a la crisis del citado grupo industrial, comparándola con la aplicada por las empresas de capital regional.
\end{abstract}

ABSTRACT. Since the final eighties the textile industry is coming into a crisis in Spain. The most aparent event in La Rioja has been the crack of the enterprises of a multinational holding. This paper describes the evolution of these firms, put in connexion with the one of the regional textil industry, emphasizing the strategy of reorganisation against the crisis and comparing it with the one of the regional firms.

Palabras-clave: La Rioja, industria textil, grupo Prouvost.

Key-words: La Rioja, textile industry, Prouvost holding.

\section{Introducción}

Cuando a principios de 1992 la prensa regional publicó la noticia de que a lo largo de ese año se iban a marchar de Logroño las dos empresas del grupo Prouvost, es decir, "Hilaturas Prouvost - Estambrera Riojana, S.A." y "Europunto, S.A.", cualquier persona que hubiera seguido con interés los avatares de la industria en las últimas décadas se tuvo que dar cuenta de que se iba a producir un acontecimiento importante en el proceso de industrialización de La Rioja: la desaparición de estas dos empresas no es, una vez consumada, uno más de los cierres o traslados de fábricas que se han producido últimamente, sino que aparece ante los ojos del observador revestida de una trascendencia especial. Esto es asi porque tanto "Estambrera" como

\footnotetext{
* Departamento de Geografia y Ordenación del Territorio, Universidad de Zaragoza.
} 
"Europunto" presentaban unos rasgos muy peculiares dentro del contexto de la industria riojana:

En primer lugar, su excepcional tamaño: "Estambrera" fue la empresa que tuvo la mayor plantilla de trabajadores de toda la historia económica regional; en La Rioja nunca ha habido ninguna que haya alcanzado los mil empleados, siendo "Estambrera" la que más se ha aproximado a esa cifra; en un contexto empresarial caracterizado por el predominio abrumador de la pequeña y mediana empresa un gran establecimiento fabril siempre es excepcional y su presencia puede ser, por ello, especialmente significativa. Su compañera de grupo, "Europunto", también ha tenido un tamaño grande en relación con el conjunto de la industria riojana, aunque su plantilla se ha mantenido siempre dentro de cifras más modestas que la otra: en algún momento llegó a superar los quinientos trabajadores, pero casi siempre ha permanecido por debajo. Cuando dos empresas excepcionales por su tamaño se marchan hay que reflexionar seriamente sobre tal hecho e intentar encontrarle una explicación clarificadora.

En segundo lugar, fueron excepcionales en otro sentido igualmente significativo: "Estambrera" fue la primera empresa de La Rioja participada por el capital extranjero después de la guerra civil; había sido fundada durante la autarquia económica por fabricantes de lana de la región y en los años sesenta el grupo textil francẽs "Prouvost" adquirió la mayoría de sus acciones. "Europunto", por su parte, fue la primera empresa creada directamente por el capital multinacional en La Rioja: una vez instalado en la región merced a la compra de "Estambrera", el mencionado grupo decidió diversificar la producción de hilados de ésta creando un nuevo establecimiento dedicado a la fabricación de géneros de punto. Tampoco puede ni debe pasar desapercibido el hecho de que desaparezcan de La Rioja las empresas que marcan el comienzo de la entrada del capital extranjero en la región.

Por otra parte, no todo en ellas es excepcional; también son, en alguna medida, representativas de la industria riojana; ambas pertenecen al sector textil y su trayectoria ha estado ligada a la marcha general del sector en La Rioja, como muestran estos dos hechos: en primer lugar, "Estambrera" fue creada en pleno periodo autárquico, cuando la industria textil regional realizó un esfuerzo de transformación importante, que convirtió a Logroño en un centro secundario, pero no desdeñable, de la industria textil lanera a nivel nacional. En segundo lugar, "Estambrera" y "Europunto" se marchan de Logroño a la vez que el conjunto del sector textil riojano entra en una aguda crisis, de la que parece que no va a salir; la desaparición de ambas no constituye en este sentido un caso aislado, aunque sea especialmente significativo por las razones de excepcionalidad antes comentadas. Por ello, el análisis de la trayectoria de estas dos empresas puede facilitar el conocimiento del sector textil riojano.

Lo que se pretende en este trabajo es, en consonancia con lo anterior, analizar la historia de dos empresas concretas ligadas entre sí para, a partir de ese análisis, comprender mejor la evoluciôn de la industria textil en La Rioja. 


\section{La evolución de la industria textil riojana hasta el plan de estabilización y la fundación de "Estambrera"}

La Rioja es una de las regiones españolas en que la Mesta estuvo fuertemente implantada: en las sierras de la Demanda y de los Cameros, correspondientes a las estribaciones más occidentales del Sistema Ibérico, hubo desde finales de la Edad Media y a lo largo de la Moderna cuantiosos rebaños de ovejas merinas, productoras de lana, que se criaban en régimen de trashumancia, de manera que pasaban el verano en las praderas de montaña y en otoño realizaban, a través de las cañadas, el camino de Extremadura o La Mancha, en cuyas dehesas pasaban el invierno. En relación con esta actividad pastoril se desarrolló una industria artesanal lanera que alcanzó su máxima expansión en el siglo XVI. A partir de entonces se inició una cierta decadencia, pero en el XVIII aún tenian las manufacturas textiles cameranas una destacada importancia en el conjunto del país.

Por otra parte, en los valles del Alhama y su afluente el Linares, donde las montañas presentan una altura sensiblemente menor $\mathrm{y}$, por tanto, peores disponibilidades de pastos para el ganado y mejores posibilidades agricolas, se desarrolló la fabricación de tejidos de lino, planta cuyo cultivo estuvo muy extendido, como indica el propio nombre del mencionado afluente.

En los años treinta del siglo pasado comenzó en Cataluña la revolución industrial, centrada precisamente en el sector textil, que se inició con la mecanización del hilado y el tejido del algodón; algo más tarde se aplicaron las mismas técnicas a la lana. Las producciones textiles industriales de Cataluña fueron conquistando poco a poco el mercado interior, arrinconando paulatinamente a la producción artesanal y sustituyendo a las mercancías importadas de otros paises. De esta manera el siglo XIX fue el siglo de la desaparición, en muchos casos definitiva, de la fabricación artesanal de paños de lana y lienzos de lino, ante la competencia de los productos industriales de lana y algodón, respectivamente, de las fábricas catalanas (NADAL, 1985).

Esto fue lo que ocurrió en muchas regiones españolas, pero en algunas los empresarios tuvieron la habilidad y el valor de enfrentarse a esa competencia con sus mismas armas y comenzaron a instalar las primeras máquinas. La Rioja es una de ellas: a partir de los años sesenta del siglo pasado se fueron mecanizando los procesos productivos artesanales en un grado suficiente como para afirmar que La Rioja no fue una región al margen de la revolución industrial, aunque, si se entiende ésta como proceso global de cambio socioeconomico, hay que reconocerle un alcance bastante limitado.

Se puede presentar una primera imagen cuantitativa del textil riojano para el año 1915, en el que se hizo un minucioso censo de empresas industriales (CONSEJO PROVINCIAL DE FOMENTO. LOGROÑO, 1915). Entonces había en La Rioja 46 empresas textiles, que daban empleo a 755 trabajadores, cifras que equivalen al $8,5 \mathrm{y}$ al 10 por ciento de los totales respectivos de la industria regional. Sin duda se trata de un modesto balance.

La localización de estas primeras empresas resulta muy significativa: la mayor concentración se daba en Logroño, con 189 trabajadores, la cuarta 
parte del total. Le seguian otras cuatro localidades, cuyos porcentajes de empleo estaban comprendidos entre el 14 y el 18 por ciento: Aguilar del Río Alhama, Cervera del Río Alhama, Enciso y Ortigosa de Cameros; con cifras inferiores, que no alcanzaban el 12 por ciento en conjunto, aparecen otras cinco localidades: Munilla, Ezcaray, Arnedillo, Haro y Viguera. Exceptuando Logroño y Haro, que se localizan a orillas del Ebro, en pleno centro de la depresión que lleva su nombre, las demás localidades se hallan ubicadas en la zona montañosa, en los valles de cuatro afluentes del río: el Oja (Ezcaray), el Iregua (Ortigosa y Viguera), el Cidacos (Arnedillo, Enciso y Munilla) y el Alhama (Aguilar y Cervera). Esta distribución espacial de principios del siglo $\mathrm{XX}$ es un fiel reflejo de la historia, puesto que las empresas se encuentran en los mismos lugares en que se localizaban los talleres artesanales de lana y lino, evidenciando asi que esta industria procede de la modernización de la actividad textil tradicional.

La evolución desde 1915 hasta mediados de siglo no ha podido ser suficientemente cuantificada, pero de la consulta de diversa bibliografia (RUIZ DE LA CUESTA, 1952, OCHAGAVIA, 1957) se desprende que la industria textil riojana debió consolidarse en los años veinte y que conoció un notable impulso durante la guerra civil. La ruptura del mercado nacional como consecuencia de la misma y el hecho de que los principales centros textiles quedaran en el territorio de la república (Cataluña sobre todo, pero también Valencia y Madrid) impulsó la industria en la otra zona: entre 1936 y 1938 se duplicó en ésta la producción de tejidos de lana y se triplicó la de tejidos de algodón (MALEFAKIS, 1987). Sin duda La Rioja, cuyos jefes militares se sumaron a la sublevación practicamente desde el primer dia, no fue ajena a este crecimiento.

Terminada la guerra se inició la época de la autarquía económica que, a juzgar por algunas cifras, resultó positiva para la industria textil regional. En 1953 destacaban nueve fábricas, siete dedicadas a los tejidos de lana y dos a los de algodón, que daban empleo a 1.240 trabajadores (CONSEJO SUPERIOR DE INDUSTRIA, 1953), pero había también numerosos talleres y pequeñas empresas, de imposible cuantificación, dedicados a la producción de géneros de punto, cuerdas, sacos, borra y regeneración de tejidos. Finalizando la década de los cincuenta se registró una notable ampliación de las empresas y una sensible mejora de las instalaciones (CONSEJO ECONOMICO SINDICAL PROVINCIAL DE LOGROÑO, 1958), aprovechando las posibilidades de equipamiento que se abrieron entonces gracias a los primeros atisbos de apertura al mercado internacional.

Cuando se aplicó el Plan de Estabilizaciôn de 1959, que castigó severamente a gran cantidad de empresas que se habían desarrollado bajo la artificiosa protección de la época autárquica, el sector textil de La Rioja se adaptó bien a la nueva situación, consolidando su posición en el mercado nacional: en 1955 se generaba en La Rioja el 0,43 por ciento del valor añadido bruto de la industria textil nacional, pero en 1962 se habia duplicado ese porcentaje, alcanzando el 0,85 por ciento (BANCO DE BILBAO, 1978). 
Este desarrollo continuo del sector textil riojano estuvo acompañado de un proceso de relocalización muy significativo: en 1920 una de las empresas de la localidad de Munilla se desdobló, mediante la construcción de una fábrica nueva en Logroño, manteniéndose la originaria en funcionamiento hasta que en 1945 fue desmantelada y trasladado su equipo funcional a la capital de la provincia. Es el primero de una serie de casos que se desarrollan siguiendo la misma secuencia: primero se crea una fábrica nueva fuera de la sierra (desdoblamiento) y luego se cierra la vieja, trasladando a la nueva lo que resulte aprovechable (CALVO PALACIOS, 1972); en otros casos las empresas cierran sin pasos intermedios. Este proceso tiene lugar a lo largo de los años veinte, treinta, cuarenta y cincuenta, culminando poco después del plan de estabilización; el balance final es la desaparición de la industria de montaña y la concentración de la mayor parte del textil regional en Logroño.

El proceso es completamente lógico: los fabricantes comprenden que no pueden competir en el mercado si se mantienen en zonas mal comunicadas, lejos del ferrocarril, con carreteras de sinuoso trazado y difícil conservación, sufriendo irregularidades en el suministro de energia eléctrica y alejados de los centros de poder, con los que hay que mantener relaciones frecuentes, sobre todo en épocas de fuerte intervención estatal, como la autarquía. Por eso a la hora de modernizar o ampliar sus instalaciones la opción más sensata a los ojos de los empresarios es realizar las inversiones en los puntos que ofrecen mayores ventajas de localización y, sin duda, Logroño es el mejor de la provincia en este sentido. En definitiva, la industria textil camerana se hace logroñesa o, por decirlo de un modo más general, la industria rural de montaña emigra a la ciudad, convirtiéndose en urbana.

En este contexto de desarrollo autárquico y relocalización surge la empresa "Estambrera Riojana, S.A.", creada en la misma ciudad de Logroño por fabricantes de lana de la provincia y dedicada a la producción de hilado de estambre. En el año 1953 tenía 45 trabajadores (CONSEJO SUPERIOR DE INDUSTRIA, 1953), siendo la más pequeña de las nueve fábricas a que antes se ha hecho referencia. Se trata, pues, de una empresa modesta en sus origenes, que se crea en una época de expansión del sector textil en La Rioja y que se localiza en Logroño, o sea, en el emplazamiento elegido por las fábricas textiles cameranas que han renunciado a mantenerse en sus lugares de origen.

\section{La época del desarrollo y la instalación del grupo Prouvost en La Rioja}

Entre las medidas relacionadas con el plan de estabilización destaca una nueva normativa sobre inversiones extranjeras, que autoriza la participación de capitales de otros países en empresas españolas a la vez que garantiza la transferencia de beneficios y la salida de aquéllos en caso de venta. En los años sesenta España resultaba un país atractivo para la inversión extranjera por disponer de un mercado interno amplio, en rápida expansión y muy pro- 
tegido, unos costes laborales relativamente bajos y una conflictividad laboral casi inexistente. Por eso entre 1959 y 1977 se produjo un importante flujo de capitales extranjeros, valorado en el 6 por ciento de la formación bruta de capital fijo realizada en el pais en esos años (MENDEZ, 1988).

Uno de los grupos financiero-industriales más importantes del sector textil en Europa es "Vitoux Etabliment Vitos-Prouvost", auténtico holding formado en torno a la "Lainière de Roubaix", empresa de la tradicional región lanera del norte de Francia; el grupo tiene su sede central en París y está constituido por un conjunto de empresas textiles y de confección establecidas en su pais de origen, en Bélgica y en Alemania ${ }^{1}$.

Dicho grupo centró su atención en España a comienzos de los años sesenta y no le pasó desapercibido el esfuerzo inversor llevado a cabo durante la autarquía por algunas de las empresas textiles de La Rioja, sintiêndose especialmente atraido por la más moderna de ellas, "Estambrera Riojana", de la que se convirtió en socio mayoritario tras la compra del correspondiente paquete de acciones.

La empresa se especializó en dos tipos de producto: hilo industrial para las fábricas de confección y géneros de punto e hilo para labores domésticas; en el primer caso se continuó utilizando la marca de antes, que no es otra que el mismo nombre de la empresa, pero en el segundo se adoptó la del grupo ("Pingoüin"), con la que se inició una fuerte penetración en el mercado nacional. Tras sucesivas ampliaciones la empresa pasó a tener 426 trabajadores en 1969 (IBERPLAN, 1971) y 612 en 1974 (BONO RIOS, 1978).

El mismo grupo creó en 1967 una empresa de géneros de punto, con el nombre de "Europunto S.A.", que se convirtió así en uno de los principales clientes de "Estambrera"; las prendas de vestir fabricadas se comercializaron con las marcas del grupo ("Rodier", "Vitos" y otras menos conocidas). En el año 1969 tenia 140 empleados (CONSEJO SUPERIOR DEL MINISTERIO DE INDUSTRIA, 1971), pero registró después varias ampliaciones hasta alcanzar una plantilla de 538 trabajadores en 1974 (BONO RIOS, 1978); el crecimiento, como se ve, fue espectacular.

Desde su base en Logroño, Prouvost llevô a cabo algunas inversiones en otros puntos del país, participando en empresas ya existentes ("Peinaje del Rîo Llobregat" o "Trilla y Bruguera", en Cataluña) y creando otras nuevas ("Euronavarra, S.A.", en la localidad navarra de Puentelarreina); asi España pasó a ser uno más del conjunto de países europeos en que operaba el grupo.

Con la incorporaciôn del capital extranjero y la dinámica claramente expansiva de las demás empresas del sector, La Rioja vio incrementada su participación en el valor añadido bruto de la industria textil nacional del 0,85 por ciento en 1962 al 0,91 en 1969 y el 0,95 en 1975 (BANCO DE BILBAO, 1978). Es importante destacar que en esos años la industria riojana, consi-

1. Los datos sobre el holding y sus empresas, cuando no se cita expresamente la fuente, proceden de la prensa y de los expedientes laborales a que se hará referencia más adelante; en el otoño de 1992 se solicitó a la dirección del grupo en Logroño información para este artículo, indicando la finalidad puramente cientifica del mismo, pero la solicitud fue contestada negativamente, alegando la situación de traslado en que se encontraban las empresas. 
derada en bloque, creció más despacio que la del conjunto del país (CLIMENT LOPEZ, 1992), por lo que la evolución de signo contrario que experimenta el textil es un indicador claro de que se trata de un sector dinámico a nivel regional. Una buena parte de ese dinamismo cabe atribuirlo a las empresas del grupo Prouvost, pero sin perder de vista la buena marcha del conjunto de las empresas regionales.

\section{La crisis industrial de 1973-1985 y su reflejo en el textil de La Rioja}

En la primera mitad de los años setenta se agota el modelo de desarrollo vigente en las décadas anteriores y comienza un largo periodo de crisis, que afecta fundamentalmente a la industria y, dentro de ella, a algunos sectores concretos, entre los que se encuentra el textil. Las causas generales de la crisis de éste a nivel mundial y, especialmente, en España son: la subida de los precios del petróleo, que implica un encarecimiento tanto de la energía como de las fibras sintéticas, las más utilizadas ya por entonces, que se elaboran a partir de derivados petroliferos; la concurrencia cada vez mayor en el mercado internacional, que obliga a competir con los productos de los llamados nuevos paises industriales del sureste asiático y de Latinoamérica, mucho más baratos por los menores costes de la mano de obra, y con los de países altamente desarrollados, arropados por marcas comerciales de prestigio; y, finalmente, la aceleración tecnológica que experimentan los procesos fabriles de hilado y tejido, que exige de las empresas un continuo esfuerzo inversor para no quedar obsoletas.

No obstante, la década de los setenta no fue para la industria textil de La Rioja una época de crisis aguda ni profunda: su participación en el valor añadido del sector a nivel nacional siguió incrementándose, pasando del 0,95 por ciento en 1975 al 1,27 en 1977 y al 1,29 en 1979 (BANCO DE BILBAO, 1978 y siguientes). En estos años continuaron incrementándose las plantillas de las empresas, como ocurrió con las del grupo Prouvost, que pasaron de 1.150 trabajadores en 1974 (BONO RIOS, 1978) a 1.461 en 1978 (METRA SEIS, 1986); dos años más tarde "Estambrera" llegó a tener 905 empleados, cifra que marca no sólo su tamaño máximo sino también el récord absoluto de La Rioja. Esta favorable evolución se explica en parte por la modernidad de las fábricas, cuyo nivel técnico era, logicamente, más alto que la media, y también por la coincidencia de la crisis con el periodo de vigencia del Polo de Desarrollo Industrial de Logroño, de cuyas ayudas se beneficiaron las principales empresas del sector, entre ellas "Estambrera" y "Europunto"2.

La gravedad de la situación del textil a nivel nacional llevó al gobierno de entonces a poner en marcha, con un notable retraso, un plan de reconversión, que estuvo vigente entre 1981 y 1986; en él se propugnaba una reducción de plantillas del 9 por ciento en el conjunto del sector, objetivo que se

2. Un análisis del Polo de Desarrollo Industrial de Logroño y su papel en la crisis económica de los años setenta, dentro de La Rioja, puede verse en CLIMENT LOPEZ (1990). 
había cumplido en la fecha prevista y que vino a resultar un ajuste bastante moderado ${ }^{3}$. También se arbitraron medidas financieras y fiscales de apoyo a las empresas, tanto para el equipamiento tecnológico como para los llamados intangibles (diseño y marcas comerciales).

Para poder acogerse a las ayudas previstas en el plan las empresas tenían que presentar ante la comisión ejecutiva del mismo un programa concreto de reconversión. Las del grupo Prouvost así lo hicieron, elaborando "Estambrera" el suyo a cinco años (1983-1987) ${ }^{4}$; en el transcurso de ese tiempo se invirtieron 1.303 millones de pesetas, el 64 por ciento de los cuales correspondió a maquinaria e instalaciones, el 10 por ciento a estudios de mercado y programas de promoción, el 9 a organización y el 7 a equipo de proceso de datos; para ello recibió una subvención estatal de 324 millones de pesetas entre 1986 y 1987 ; esto vino acompañado de una disminución de plantillas bastante moderada: en 1987 tenía 808 trabajadores (GOBIERNO DE LA RIOJA, 1988), frente a los 905 de 1980.

Otras empresas textiles riojanas se acogieron también al plan de reconversión, como "Europunto", que pasó a 428 trabajadores en 1987 (GOBIERNO DE LA RIOJA, 1988), cuando en 1974 tenia 538, y "Tricots Fajardo", que despidió a 8 trabajadores entre 1984 y 1985, quedando con una plantilla de 22 en 1986 (GOBIERNO DE LA RIOJA, 1987). Estas debieron ser las que, en proporción, más redujeron sus plantillas.

Considerado globalmente, el sector textil de La Rioja a lo largo de los años setenta experimentó una reducción de empleos más próxima a la de "Estambrera" que a la de las citadas anteriormente; fue en conjunto bastante moderada, en torno al 10 por ciento, cifra comparable a la del conjunto nacional.

\section{La crisis textil reciente y la desaparición de las empresas del grupo Prouvost.}

Algunos de los sectores afectados por la politica de reconversión industrial han superado la crisis y están en una situación saneada y progresiva o, al menos, de estabilidad; pero otros vuelven a encontrarse mal y exigen de nuevo medidas de ajuste drásticas, por lo que bien puede hablarse de fracaso de la reconversión ${ }^{5}$. El textil es uno de ellos: desde 1988 su producción a nivel nacional presenta tasas de crecimiento negativas, en contraste con el conjunto de la industria.

3. Al menos en comparación con otros sectores, que han experimentado reducciones de plantilla de más del 50 por ciento, como los de construcción naval, aceros especiales, electrodomésticos de linea blanca o siderurgia integral. El sector textil es, de todos los sometidos a planes de reconversión, el que menos excedentes de plantilla ha tenido.

4. La cifra procede de la documentación económica aportada por la empresa a los expedientes de que luego se hablará; la representación de los trabajadores estimaba en 450 millones de pesetas las subvenciones recibidas hasta 1986.

5. El ejemplo más palmario de éstos es la siderurgia integral, a la que se está aplicando otra drástica reducción de capacidad productiva, con nuevas y costosas inversiones, a las que tiene que hacer frente el Estado porque el capital privado hace ya tiempo que abandonó las empresas. 
Este hecho debe ponerse en relación con otro: hasta 1986 el sector presentó un saldo comercial positivo con el resto del mundo, aunque en descenso continuo, mientras que a partir de 1987 fue negativo; dicho de otra manera, es la competencia internacional de nuevo la que lo lleva a la crisis, sin que ahora tenga nada que ver el precio del petróleo, que a lo largo de los años ochenta ha evolucionado favorablemente para los intereses de los consumidores. Ese incremento de la competencia en estos años está directamente relacionado con el ingreso en la Comunidad Económica Europea: el acta de adhesión obligó a España, por una parte, a suprimir los contingentes a la importación de productos textiles procedentes de los restantes países miembros y a rebajar los altos aranceles que mantenía frente a ellos; por otra parte, a adoptar la tarifa exterior común frente a terceros de la Comunidad, que era sensiblemente más baja que el arancel anterior, asi como a asumir las ventajas que la CEE tenía acordadas con otros paises, especialmente con algunos del tercer mundo.

$\mathrm{E} 1$ textil es uno de los sectores industriales maduros, es decir, de los que producen bienes de elevado consumo, con sistemas de producción estandarizados, tecnologias bien conocidas y mano de obra abundante; por ello la competencia, en un mercado internacional cada vez más abierto, es muy fuerte: son muchos los países del tercer mundo en que se ha desarrollado con fuerza la industria textil, beneficiándose de unos costes salariales bajos en comparación con los españoles. Las empresas multinacionales actúan como impulsoras de ese desarrollo y de la incorporación progresiva de nuevos productores al mercado internacional, estableciendo fábricas propias en nuevos países o subcontratando con empresas locales la fabricación de ciertos productos o componentes.

Los saldos comerciales negativos y el descenso en la producción tienen su correlato en el cierre de empresas: en la principal región textil de España, Cataluña, se presentaron, sôlo en el año 1991, 274 expedientes de regulación de empleo, que afectaron a casi nueve mil trabajadores.

Los resultados de las empresas del grupo Prouvost en La Rioja durante los últimos años han ido acordes con esa evolución negativa: "Estambrera" obtuvo doscientos millones de pesetas de beneficios en 1986, pero en 1987 tuvo pérdidas por valor de 226, que llegaron en 1988 a 1.553, fiel reflejo de una caída del volumen de ventas del 45 por ciento en esos tres años. "Europunto", por su parte, arrastraba pérdidas desde 1986, con cifras anuales comprendidas entre los 276 millones de ese año y los 1.205 de 1988 . Aunque no se tienen cifras concretas, otras empresas textiles regionales experimentaron también reducciones en su volumen de ventas, deterioro de sus beneficios y, finalmente, pérdidas.

Para hacer frente a la crisis el grupo Prouvost inició una politica de reestructuración de la que se saben dos cosas: su existencia, dada a conocer por él mismo, y la manera en que afectó a las empresas instaladas en La Rioja. La prensa económica nacional se hizo eco de lo primero en los siguientes términos: V.E.V.-Prouvost "está llevando a cabo una reorganización total de su holding. El objetivo propuesto es tener reestructurada toda la sociedad antes 


\section{E.A. CLIMENT LOPEZ}

del año 1992. Para ello centralizará todos sus pedidos, tanto nacionales como internacionales, en París, sede social de la empresa. Desde allí se reorganizará la producción de la forma más rentable" (Cinco Dias, 13 de Abril de 1989).

En 1988 se producen algunos reajustes en la propiedad de las empresas españolas del grupo, entre los que cabe destacar la absorción de "Euronavarra" por "Europunto"6 . Pero esto no es lo que interesa destacar en este trabajo, sino la evolución que desde entonces van experimentando las fábricas respecto a actividades económicas y plantillas.

La dirección del grupo comenzó a aplicar una política de bajas incentivadas a lo largo de 1988, más intensa en "Estambrera" que en "Europunto", a pesar de que ésta habia tenido pérdidas más cuantiosas y durante más tiempo; a los trabajadores se les ofrecieron indemnizaciones altas a la vez que se esgrimía la posibilidad de un expediente de regulación de empleo si no se alcanzaba un número de bajas voluntarias suficiente; éste se obtuvo y a finales de año la empresa quedó con una plantilla de 613 trabajadores.

Pero, a pesar de ello, a principios de 1989 "Estambrera" inició un expediente para la rescisión de contratos a 177 trabajadores, basándose en que la situación del textil era de crisis estructural, a la que sólo cabía responder con una reducción de capacidad productiva ${ }^{7}$. En la memoria justificativa se destacaba la fuerte caída de las ventas, atribuida a: una causa de orden sociológico, el abandono de la tradicional costumbre de "hacer punto" por parte de las mujeres españolas; otra de tipo comercial, la importación de hilos industriales del tercer mundo; y una tercera de tipo técnico, la instalación masiva de máquinas electrónicas para fabricar géneros de punto, cuyo resultado es la caída de los precios de éstos, de modo que las prendas confeccionadas a menudo resultan más baratas que los ovillos de lana con que tejer dichas prendas en casa. Se señala también que la crisis es general a escala europea, por lo que las reducciones de plantilla se han producido también en otras empresas del grupo ubicadas en otros paises.

Los representantes de los trabajadores solicitaron la retirada del expediente y presentaron un contra-informe centrado en varias cuestiones: por una parte, señalaban que la empresa no parecía tener otro objetivo que la reducción de plantilla, puesto que no explicaba las medidas financieras, tecnológicas y comerciales a adoptar para hacer frente a la crisis supuestamente estructural, si se exceptúa una inversión de 200 millones de pesetas, cifra absolutamente insuficiente para cualquier proyecto serio de una gran empresa; por otra parte, atribuian las pérdidas a una situación coyuntural deriva$\mathrm{da}$, al menos en parte, de una mala gestión anterior ${ }^{8}$; finalmente, señalaban algunas contradicciones, como que la empresa se proponía, en la justificación

6. Hay otro reajuste, al menos, en Cataluña, donde el grupo vendiô su participación en "Peinaje del Rio Llobregat".

7. La consulta de dichos expedientes ha sido facilitada por la unión regional de Comisiones Obreras de La Rioja y por algunas personas del mundo sindical relacionadas en su momento con las empresas del grupo Prouvost. Quede aquí constancia de nuestro agradecimiento por su ayuda.

8. En 1986 y 1987, según el comité de empresa, se contrató, respectivamente, a 190 y 167 trabajadores eventuales, con lo que llegó a trabajarse incluso sábados y domingos; esto provocó la acumulación de grandes stocks. 
del expediente, mantener una cierta sobre-capacidad para adaptarse a las variaciones de la demanda a la vez que preveía la caida continua de la nisma, o que se programaba una reducción de la gama de hilos industriales a producir en la fábrica a la vez que la importación, para comercializar en España, de algunas otras clases producidas en Francia.

La autoridad laboral dio la razón en parte a los trabajadores, dene gando la rescisión de contratos, pero autorizó una suspensión de los mismos durante cinco meses, con el fin de que la empresa tuviera un margen de tiempo adecuado para reducir pérdidas y afrontar mejor la situación.

No obstante, cuando se cumplió el plazo de la suspensión, en el otoño de 1989, la empresa dio vacaciones a los que debían incorporarse y solicitó de nuevo la rescisión de sus contratos; la representación obrera volvió a oponerse, alegando que la empresa no aportaba elementos de juicio nuevos respecto a la petición anterior. Pero esta vez la autoridad laboral se pronunció a favor de la rescisión definitiva. De este modo, en 1989 "Estambrera" pasó a tener 430 empleados, menos de la mitad que en 1980.

El empeño de la empresa en conseguir esta reducción de plantilla indica que se trata de un objetivo importante en su estrategia de reestructuración, por lo que conviene fijarse en cómo quedó organizada la empresa después de la misma: todos los despedidos formaban parte de las secciones de producción, pero no de las administrativas ni comerciales, por lo que la proporción entre puestos de trabajo directos e indirectos quedó en 38 por 62 , mientras que en 1980 era de 64 por 36. Con esto empieza a cobrar cuerpo la hipótesis de que la dirección del grupo Prouvost ha optado por liquidar la producción de la fábrica y convertir a "Estambrera" en una empresa puramente comercializadora.

La postura de rechazo por parte de los trabajadores, combativa en vez de negociadora, se basaba precisamente en la sospecha de que ésa era la estrategia del grupo y que a esos dos expedientes seguirian otros hasta la liquidación de la empresa: no se trataba, por tanto, de conseguir mejores indemnizaciones para los 177 trabajadores afectados en ese momento ni de negociar esa cifra en base a un plan de futuro, sino de evitar la destrucción de esos puestos de trabajo y, a la vez, garantizar la continuidad de todos los demás. La resolución de la autoridad laboral pudo interpretarse, en este sentido, como una amarga derrota y trajo consigo el descrédito de las actitudes combativas.

En noviembre de 1990 la dirección de la empresa presentó un tercer expediente de rescisión de contratos, a pesar de que el director general había declarado en mayo a la prensa que no tenía intención de presentar ningún otro. Afectó nada menos que a 302 trabajadores, todos ellos pertenecientes a las secciones de producción, que de este modo fueron completamente desmanteladas, quedando en funcionamiento únicamente las de comercialización. La representación de los trabajadores en este caso no se opuso al expediente, orientándose a conseguir indemnizaciones superiores a las establecidas por la legislación laboral. La línea de fabricación se cerró en enero de 1991, fecha a partir de la cual "Estambrera" dejó de ser una empresa indus- 
trial, para dedicarse exclusivamente a la comercialización de los productos que, con las marcas registradas del grupo Prouvost, elaboran otras empresas pertenecientes al mismo o ligadas a él mediante contrato. Existe la convicción de que algunas de estas empresas están localizadas en países de costes laborales más bajos ${ }^{9}$.

Una vez encauzada la liquidación de "Estambrera", le toca el turno a "Europunto": en 1990 se procede al cierre del centro productivo de Puentelarreina (Navarra), acompañado de una política de bajas incentivadas en el de Logroño, que se prolonga durante el año siguiente.

A principios de 1992 la dirección de la empresa presenta un expediente de regulación de empleo, solicitando la extinción de relaciones laborales con 297 de sus 387 trabajadores, lo cual significa, igual que en el caso de su compañera de grupo, desmantelar las actividades de fabricación y mantener sólo las de comercialización. La memoria justificativa se limita a señalar la existencia de pérdidas, sin un análisis riguroso de la causa de las mismas y sin apuntar medidas para salir de la situación; los trabajadores no se oponen, limitándose a negociar el monto total de indemnizaciones

Un poco más adelante tiene lugar el desenlace definitivo: las dos empresas presentan sendos expedientes de movilidad geográfica para trasladarse a Barcelona, alegando razones de estrategia comercial, pues la capital catalana aventaja a Logroño en infraestructuras de transporte y comunicaciones, cuenta con numerosas empresas y profesionales del diseño, el marketing o la publicidad $\mathrm{y}$, al tratarse de uno de los principales centros europeos de la moda, es fácil realizar en ella contactos a través de los cuales se puede obtener información valiosa. El expediente es aprobado por la administración, ofreciéndose a los trabajadores la posibilidad de seguir en las empresas, trasladándose a Barcelona o, caso de no desear el traslado, recibir una indemnización. Con esto el grupo Prouvost abandona La Rioja después de tres décadas de presencia en ella.

No son éstas las únicas empresas que han desaparecido del panorama industrial textil riojano. Destaca el caso de "Textil Quemada", una de las más antiguas de la región ${ }^{10}$, dedicada tambiên a la producción de lana: en diciembre de 1989, tras varios años de pérdidas y endeudamiento, solicitó la suspensión temporal de los contratos de 112 de sus 116 empleados, para obtener un respiro. Mientras duró aquélla la dirección proclamó su confianza en la viabilidad de la empresa, indicando la existencia de pedidos suficientes y presentando su crisis ante todo como una cuestión de liquidez; pero no convenció a las entidades financieras ni obtuvo ayuda del gobierno regional, al que

9. Al no haber recibido información directa de la empresa no se tiene completa certeza respecto a cuáles son los paises en los que se elaboran los productos que comercializan "Estambrera" y "Europunto", pero hay indicios que apuntan, por una parte, a Portugal, que a sus salarios inferiores une la condición de miembro de la Comunidad Económica Europea, y por otra a Brasil y Marruecos, paises emergentes en el panorama textil internacional.

10. "Textil Guemada" aparece atestiguada como fábrica de paños desde 1752 , con domicilio en la localidad camerana de Enciso (RUIZ DE LA CUESTA, 1952); en 1939 se desdobló, estableciendo fábrica en Logroño y manteniendo la originaria hasta finales de los años cincuenta, en que fue definitivamente desmantelada. 
recurrió en última instancia; la empresa fue definitivamente cerrada en 1990, con lo que dieron fin más de dos siglos de historia. Otro caso más reciente ha sido el de "Punto Richards", que tambiên ha cerrado sus puertas..

El conjunto del sector textil riojano ha pasado de tener 2.617 empleos en 1987 a 1.619 en 1990 (GOBIERNO DE LA RIOJA, 1988 y 1991); el de la confección ha crecido ligeramente, de 612 a 671 , incremento que de ninguna manera compensa la pérdida del otro, que se cifra en casi mil trabajadores o, en términos relativos, el 38 por ciento, en sólo tres años. Pero desde 1990 aún se han perdido al menos otros 601 puestos de trabajo textiles, los de las empresas a cuyo cierre o traslado se ha hecho referencia ${ }^{11}$, con lo que quedan como mucho 1.018, el 39 por ciento de los de 1987.

Con esto parece cerrarse un ciclo: el textil, que fue un sector artesano de gran importancia en la etapa pre-industrial de la región y uno de los primeros en incorporar la maquinaria y métodos de trabajo propios de la industria moderna, ha quedado reducido a una situación casi marginal en la estructura productiva de La Rioja.

\section{La reestructuración de la industria textil y la estrategia del capital multinacional}

La cita periodistica recogida antes afirmaba que el grupo Prouvost tenia previsto reorganizar la producción de sus empresas "de la manera más rentable"; lo analizado en el apartado anterior permite entender cómo ha enfocado el grupo la cuestión de la rentabilidad: las tareas de fabricación, que requieren mano de obra relativamente abundante y tecnologia convencional, han sido desplazadas hacia países con costes laborales bajos, con lo que se abarata la producción, mientras que las tareas relacionadas con la comercialización (incluyendo entre ellas el diseño, la creación de marcas y la publicidad) se mantienen en los países desarrollados, donde se puede disponer de mejor información y encontrar el apoyo logistico de profesionales expertos y de empresas especializadas en servicios avanzados.

Esta estrategia ha sido utilizada sistemáticamente por las empresas multinacionales de los sectores maduros: la reducción de los costes productivos ha sido siempre uno de los objetivos a alcanzar, pero la progresiva mundialización de los intercambios comerciales ha hecho de la misma una necesidad imperiosa en las últimas décadas. Dicha reducción puede conseguirse utilizando mano de obra más barata, sin necesidad de disminuir las plantillas, o aumentando la productividad de la mano de obra, lo cual requiere mejorar la relación capital-trabajo con fuertes inversiones; las empresas escogen entre ambas opciones o combinan una y otra en función de sus posibilidades. ción.

11. Algunas empresas han reducido sus plantillas, aunque se ignora la cuantia de la reduc- 
El proceso reciente de las dos empresas del grupo Prouvost muestra que éste ha optado claramente por trabajar con mano de obra más barata y que La Rioja ya no tiene el atractivo que, en este sentido, tenia cuando decidió instalarse en la región. Lo malo es que tampoco tiene la suficiente infraestructura de servicios avanzados como para propiciar el mantenimiento de las tareas de comercialización. En definitiva, podria decirse, usando los términos de la teoria centro-periferia, que la estrategia del grupo multinacional ante la crisis del sector textil ha consistido en trasladar las actividades de fabricación a lugares que ocupan una posición más periférica que La Rioja en el sistema económico mundial y las de comercialización a lugares más centrales.

Las empresas de capital regional, que son de tamaño modesto y no cuentan con el respaldo de un grupo financiero-industrial, no han podido aplicar la misma estrategia para salir de la crisis. Algunas de ellas han fracasado totalmente, como "Textil Guemada", a cuya desaparición se ha hecho referencia. Pero otras, de menor tamaño y sobre todo entre las dedicadas a la confección, han centrado tambiên su respuesta a la crisis exclusivamente en la reducción de los costes laborales, aplicando fórmulas que a su condición de poco ortodoxas añaden la de encontrarse bastante extendidas:

Una de ellas consiste en pactar el despido con un cierto número de trabajadores, a los que después se encarga la realización de trabajo a domicilio, facilitándole la empresa los medios de producción; de este modo, aun cuando el trabajador reciba la misma remuneración que antes por su trabajo, disminuyen los costes laborales al desaparecer las cotizaciones a la seguridad social y, además, el empresario gana en flexibilidad, pudiendo ajustar instantáneamente el volumen de encargos a la demanda y dar por terminada la relación si el negocio va mal; la flexibilidad es muy importante en un sector que depende estrechamente de algo tan fluctuante como la moda. Esto constituye para el trabajador un auténtico paso a la clandestinidad, si no formaliza su nueva situación como autónomo, y el deterioro de sus condiciones de trabajo aunque lo haga, pues a la mayor inseguridad de sus ingresos debe añadir mayores cotizaciones e impuestos; pero siempre es un mal menor en comparación con el cierre definitivo a que puede recurrir la empresa si no se acepta una propuesta de este tipo ${ }^{12}$.

En algunas ocasiones, después del cierre de una empresa los antiguos trabajadores se han visto sorprendidos por el hecho de que el empresario ha abierto una empresa nueva dedicada a las mismas o parecidas tareas que la que cerró. Este falso cierre es también una forma de abaratar los costes laborales: los nuevos trabajadores cobran un salario inferior, aunque sólo sea porque no tienen antigüedad ${ }^{13}, \mathrm{y}$ además los empresarios se acogen a los diversos incentivos vigentes para la creación de puestos de trabajo, que normalmente son exenciones o reducciones de la cuota empresarial a la seguridad social;

12. Puede haber tambiên fraude a la Seguridad Social si el trabajador percibe el seguro de desempleo a la vez que realiza dicho trabajo.

13. Aunque sean los mismos, circunstancia relativamente frecuente puesto que los empresarios a menudo buscan a los trabajadores de la antigua empresa que consideran más adecuados. 
la legislación, por otra parte, permite la generalización de contratos temporales, con lo que el empresario puede organizar la fabricación de manera más flexible que antes. Para el trabajador esto significa el deterioro de sus condiciones laborales, pues pasan a una situación de precariedad en el empleo, utilizando la terminologia acuñada por los sindicatos.

Estas dos formas de reducir costes laborales no constituyen en realidad soluciones a la crisis; son más bien salidas de emergencia puramente temporales. Aunque con ellas se consiga abaratar el producto siempre habrá países con salarios más bajos: si hace años los artículos textiles "made in Taiwan" resultaban muy competitivos por la baratura de la mano de obra, ahora resulta que en Filipinas o Tailandia se están pagando sueldos varias veces inferiores a los de aquel pais. Nunca será posible competir en este terreno, pero aunque lo fuera no sería deseable: después de los esfuerzos que ha realizado la población española en las últimas décadas para alcanzar los niveles de desarrollo económico y bienestar actuales nadie se puede plantear como objetivo que los salarios en la industria textil ni en ningún otro sector se equiparen a los de paises de menor grado de desarrollo, porque ello implicaría una caída importante del nivel de vida.

Con las medidas descritas se consigue la flexibilidad de plantillas necesaria para adaptarse con rapidez a las oscilaciones de la demanda, lo cual contribuye también a la reducción de costes, pero cabe preguntarse si no habría fórmulas con las que obtener los mismos resultados evitando la clandestinidad y la precariedad del empleo. Los empresarios dicen que esto se conseguiría eliminando las rigideces del mercado laboral y reduciendo al mínimo la intervención administrativa en todo lo relacionado con el despido de trabajadores; éstos, por el contrario, valoran como conquistas sociales algunas de las normas que los empresarios llaman rigideces y consideran la intervención administrativa como una cierta garantía frente a la capacidad de decisión absoluta de éstos. La clave del asunto está en armonizar el legitimo deseo de bienestar y seguridad de los trabajadores con la necesaria capacidad de maniobra de los empresarios en un mercado cada vez más amplio y más competitivo; cómo conseguirlo es una de las cuestiones más importantes a debatir y resolver en España en estos momentos.

Pero es que, además, hay otras estrategias de respuesta a la crisis: las que se basan en conseguir una mayor calidad de los productos y un diseño más esmerado, así como la mejora tecnológica del equipo productivo, terrenos en los que los paises menos desarrollados no pueden competir. La cuestión es si los empresarios españoles y, dentro de ellos, los riojanos, están capacitados para desarrollar este tipo de estrategias; de no estarlo a corto plazo el textil desaparecerá de la región y del país, dada la imposibilidad de competir a base de costes laborales bajos.

Los poderes públicos tienen aquí un campo de actuación adecuado: a través de los incentivos regionales, o por los medios que estimen oportunos, podrian prestar su apoyo a las empresas para la mejora de la calidad, el diseño y la innovación tecnológica; con ello resultarian más competitivas, a la vez que los trabajadores podrian tener mayor seguridad en el empleo manteniendo su nivel de vida. 


\section{Referencias bibliogrâficas:}

BANCO DE BILBAO (1978 y ss.): Renta nacional de España y su distribución provincial. Banco de Bilbao, Bilbao.

BONO RIOS, F. (1978): Tablas input-output y cuentas regionales de la economía riojana. Año 1974. Caja de Ahorros de Zaragoza, Aragón y Rioja, Zaragoza.

CALVO PALACIOS, J.L. (1972): Tres momentos en el proceso de industrialización de la provincia de Logroño. Berceo, nº 83, p. 263-281.

CLIMENT LOPEZ, E.A. (1990): El Polo de Desarrollo Industrial de Logroño. Berceo, $\mathrm{n}^{\circ}$ 118-119, p. $75-82$

CLIMENT LOPEZ, E.A. (1992): El proceso de industrialización de La Rioja. Instituto de Estudios Riojanos, Logroño.

CONSEJO ECONOMICO SINDICAL PROVINCIAL DE LOGROÑO (1958): I Pleno del Consejo Económico Sindical. Diciembre 1957. Consejo Económico Sindical, Logroño.

CONSEJO PROVINCIAL DE FOMENTO. LOGROÑO (1915): Estadistica industrial de la provincia. Imprenta y Libreria Moderna, Logroño.

CONSEJO SUPERIOR DE INDUSTRIA (1953): Momento actual de la industria en España. Provincias de Navarra y Logroño. Ministerio de Industria, Madrid.

CONSEJO SUPERIOR DEL MINISTERIO DE INDUSTRIA (1971): Memoria. Ministerio de Industria, Madrid.

GOBIERNO DE LA RIOJA (1987): Catálogo de la industria riojana. Gobierno de La Rioja, Logroño.

IBERPLAN (1971): Estudio socioeconómico de la provincia de Logroño. Logroño, (inédito).

MALEFAKIS, E. (1987): La economía española y la guerra civil. La economia española en el siglo XX. Una perspectiva histórica. Ariel, Barcelona.

MENDEZ GUTIERREZ DEL VALLE, R. (1988): Las actividades industriales. Sintesis, Madrid.

METRA-SEIS (1986): Estudio sobre el sector del calzado en La Rioja. Logroño, (inédito).

NADAL, J. (1984): El fracaso de la revolución industrial en España, 1814-1913. Ariel, Barcelona.

OCHAGAVIA FERNANDEZ, D. (1957): Historia textil riojana. Instituto de Estudios Riojanos, Logroño.

RUIZ DE LA CUESTA, C. (1952): Bicentenario de una gran industria nacional. Rioja Industrial, p. 61-65. 